\title{
Charge order in Kondo lattice systems
}

\author{
Robert Peters, , 因 Shintaro Hoshino, ${ }^{2}$ Norio Kawakami, ${ }^{1}$ Junya Otsuki, ${ }^{2,3}$ and Yoshio Kuramoto ${ }^{2}$ \\ ${ }^{1}$ Department of Physics, Kyoto University, Kyoto 606-8502, Japan \\ ${ }^{2}$ Department of Physics, Tohoku University, Sendai 980-8578, Japan \\ ${ }^{3}$ Theoretical Physics III, Center for Electronic Correlations and Magnetism, \\ Institute of Physics, University of Augsburg, D-86135 Augsburg, Germany
}

(Dated: April 3, 2019)

\begin{abstract}
Charge order is a commonly observed phenomenon in strongly correlated materials. However, most theories are based on a repulsive inter-site Coulomb interaction in order to explain charge order. We here show that only due to local interactions, charge order is favorable in heavy fermion systems at quarter filling. The driving force is the Kondo effect, which leads to a non-linear dependence on the filling of the lattice site for the energy gain. We use dynamical mean field theory combined with numerical renormalization group to demonstrate the existence of charge order at quarter filling for a Kondo lattice model.
\end{abstract}

PACS numbers: 71.10.Fd;71.27.+a;75.25.Dk

\section{INTRODUCTION}

Heavy fermions are one of the most studied materials in condensed matter physics. This is due to a large number of intriguing phenomena ranging from quantum criticality to unconventional superconductivity, which can be found in these systems ${ }^{1-3}$ Many of these phenomena are caused by a competition between the Kondo effect and long range order, as visualized in the Doniach phase diagram. $\stackrel{4}{ }$ Most of the long-range ordered phases, which have been studied theoretically so far in these materials, are magnetically ordered phases like antiferromagnetism or ferromagnetism. However, besides these phases charge order has also been found experimentally in heavy fermion systems, e.g. in $\mathrm{R}_{5} \mathrm{Ir}_{4} \mathrm{Si}_{10}(\mathrm{R}=\mathrm{Lu}$ or $\mathrm{Yb}) \stackrel{5}{\underline{5}}$ These compounds exhibit a charge density wave (CDW) at high temperatures, and a cooperation between the CDW and a magnetic or superconducting state at much lower temperatures. Charge order can be naturally expected in systems at quarter filling for strong enough repulsive inter-site Coulomb interaction. This repulsive inter-site Coulomb interaction will lead to a situation, where it is less favorable for electrons to occupy neighboring sites equally than forming a CDW. The formation of a CDW thus minimizes the effect of the repulsive inter-site interaction. A similar effect can be observed by introducing a repulsive $c$ - $f$-electron interaction in heavy fermion models.

We will show here that it is possible to stabilize a CDW in the Kondo lattice model without any inter-site Coulomb interaction. As for many other interesting effects in heavy fermion systems, the driving force is the Kondo effect. Due to the exponential dependence of the energy, which is gained by the Kondo effect, it can be energetically favorable for the system to form a CDW. The possibility of a CDW in the Kondo lattice model was already proposed by Hirsch ${ }^{6}$ in a strong-coupling expansion, who showed that perturbation theory creates an effective repulsive inter-site interaction, which may lead to a CDW phase. Recently, Otsuki et al. $\stackrel{7}{ }$ have shown that the static susceptibility for a CDW diverges at quarter filling for small coupling strengths in the Kondo lattice model, indicating the existence of a CDW. Furthermore, phases with cooperation of spin and charge order have been reported for heavy fermion models on geometrically frustrated lattices, and are known as partial Kondo screened phases. ${ }^{8-12}$ In these cases, some sites in the unit cell form a magnetically ordered state, while others stay magnetically disordered, but may have a different occupation number.

The purpose of this paper is to show that a pure CDW can be obtained at quarter filling without frustration under the assumption of a bipartite lattice, and determine the physical properties of this phase. Furthermore, we will propose an explanation for the stability of a CDW in terms of the Kondo effect.

For studying the CDW in heavy fermions, we perform calculations for the Kondo lattice model. The Kondo lattice model describes a lattice of local moments which are coupled to itinerant electrons. The Hamiltonian can be written as $4,13,14$

$$
H=t \sum_{<i, j>\sigma} c_{i \sigma}^{\dagger} c_{j \sigma}+J \sum_{i} \vec{S}_{i} \cdot \vec{s}_{i},
$$

where the first term describes electron hopping on a lattice and the second term the spin-spin interaction between a localized moment $\vec{S}$ and the spin of a conduction electron, $\vec{s}_{i}=c_{i \rho}^{\dagger} \vec{\sigma}_{\rho \rho^{\prime}} c_{i \rho^{\prime}}$ (with Pauli matrices $\vec{\sigma}$ ). Throughout the paper we will assume an antiferromagnetic coupling, $J>0$, between the conduction electrons and the localized spins, which is appropriate to describe heavy fermion systems.

For solving the Kondo lattice model we use the single site dynamical mean field theory (DMFT)!15,16 DMFT relates the lattice model to a quantum impurity model, which must be solved self-consistently. As a consequence of this mapping onto an impurity model, the momentum dependence of the self-energy in the obtained solution is neglected. This approximation becomes exact in infinite dimensions. However, comparison to experiments 
has shown that for three dimensional systems DMFT represents a good approximation, which is able to explain at least qualitatively many effects seen in real experiments. To solve the effective quantum impurity model, we use the numerical renormalization group (NRG) 17,18 NRG is able to accurately calculate spectral functions for arbitrary parameters and wide range of temperatures,$\underline{19,20}$ and is also able to resolve exponentially small energy scales, which might emerge in quantum impurity models.

This paper is organized as follows: In the next section, we will show the existence of the CDW state at quarter filling by analyzing solutions without magnetic order. This will allow us to directly study the properties of the charge ordered state. In the third section, we allow magnetic and charge order at quarter filling simultaneously, and analyze the cooperation/competition between these two forms of long range order.

\section{PARAMAGNETIC CHARGE-DENSITY-WAVE AT QUARTER FILLING}

The phase diagram of the Kondo lattice model within DMFT has been investigated by a number of authors before ${ }^{7,21}-28$ Around quarter filling, most previous studies showed the existence of a magnetically long range ordered state. However, recent quantum Monte Carlo calculations of static susceptibilities by Otsuki et al. $\underline{\underline{7}}$ have shown the divergence of the CDW susceptibility around quarter filling.

In order to go beyond the study of static susceptibilities and to investigate the properties of the charge ordered state at quarter filling, we perform calculations for an infinite dimensional Bethe lattice with bandwidth $W$ and DOS $\rho(\omega)=8 /\left(\pi W^{2}\right) \sqrt{W^{2} / 4-\omega^{2}}$. Furthermore, we divide the lattice into two sublattices, from now on called A- and B-sublattice. In order to calculate the CDW state, we initialize these two sublattices with different chemical potentials in the first DMFT iteration, and then iteratively solve the DMFT self-consistency equation, using the same chemical potential for both lattice sites. Furthermore, breaking the spin symmetry by applying a magnetic field in the first DMFT iteration will allow to study combinations of charge order and magnetism. Preserving the spin symmetry will lead to a paramagnetic state.

In this section, we neglect magnetically ordered states. We show in Fig. 1 that a CDW exists for the Kondo lattice model even without inter-site repulsive Coulomb interaction. All solutions exhibiting charge order, which are found in this study, are located in the vicinity of quarter filling, $\left\langle n_{A}\right\rangle+\left\langle n_{B}\right\rangle=1$. In Fig. 1 we show the charge order parameter $\Delta n=\left|\left\langle n_{A}\right\rangle-\left\langle n_{B}\right\rangle\right|$ for different temperatures and coupling strengths. Furthermore, a phase diagram plotting the amplitude of the CDW over temperature and coupling strength is shown in Fig. 2.

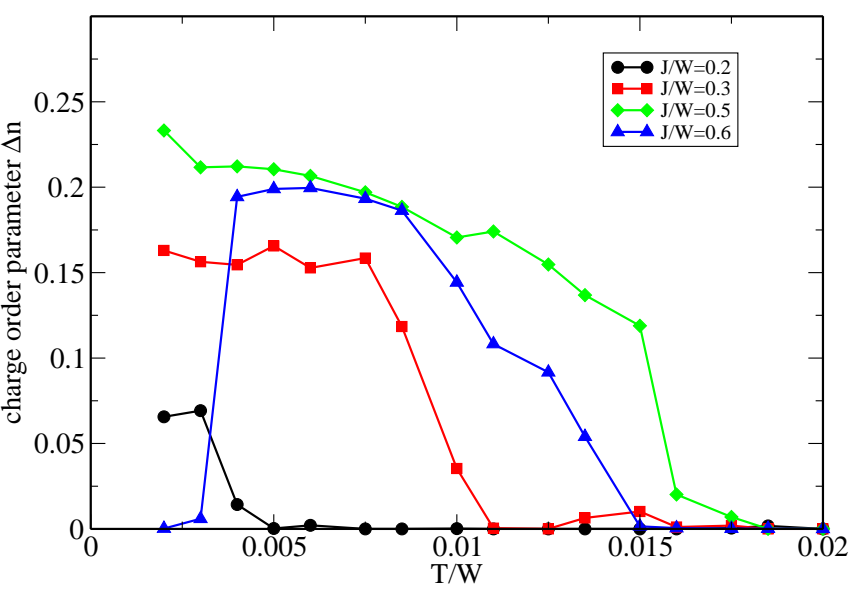

Figure 1: (Color online) Charge order parameter for a paramagnetic state at quarter filling for different temperatures and coupling strengths $J$. Lines are a guide for the eye.

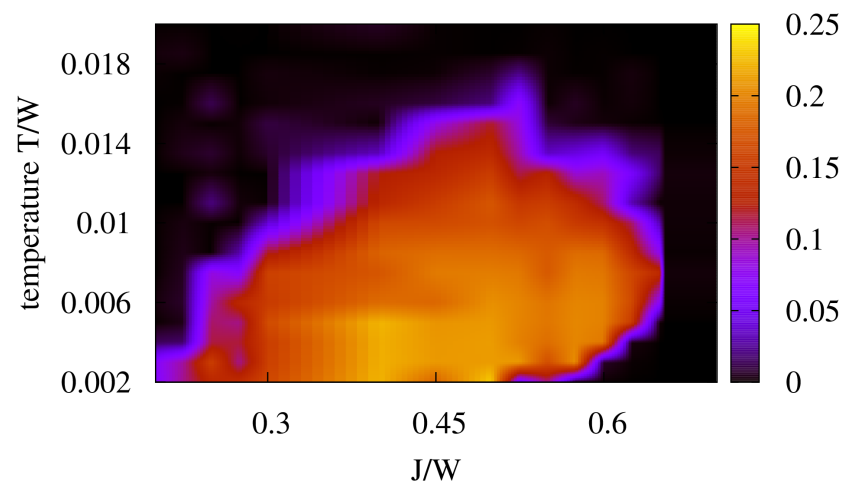

Figure 2: (Color online) Magnitude of the charge order parameter for different temperatures and coupling strengths.

Increasing the coupling strength $J$, the transition temperature as well as the charge order parameter itself increases. The magnitude of the charge order reaches a maximum of $\left|n_{A}-n_{B}\right| \approx 0.23$ for $J \approx 0.5 \mathrm{~W}$. Thus, the less occupied lattice sites are even at $T=0$ filled by $\langle n\rangle \approx 0.38$. At weak coupling strengths, $J / W<0.3$, the transition temperature increases rapidly with increasing coupling strength. Such a rapid change in the transition temperature could be explained by the exponential dependence of the Kondo effect on the coupling strength. Further evidence that the Kondo effect is essential for stabilizing charge order at quarter filling is that we find no sign of charge order for the ferromagnetically coupled Kondo lattice model, $J<0$, for which the Kondo effect is not present.

At a coupling strength $J \approx(1 / 2) W$, the behavior changes dramatically. The transition temperature and the amplitude of the order parameter begin to decrease. More importantly, charge order seems to disappear at $T=0$. For coupling strengths $0.5 W<J<0.65 W$ we find a reentrant behavior (homogeneous - charge order - 
homogeneous) for decreasing the temperature. Eventually, for $J \approx 0.65 W$ charge order vanishes completely for all temperatures.

To clarify the behavior at strong coupling strengths in more detail, we show in Fig. 3 the order parameter at fixed temperature in the lower panel and the region of chemical potential, for which a charge ordered state can be found at quarter filling, in the upper panel. It is evident from Fig. 3 that the width of the parameter region, in which the CDW can be found, decreases with increasing coupling strength. The amplitude of the charge order within this region is only slightly changed when increasing the coupling strength. However, at a critical coupling strength the parameter region vanishes completely. This behavior indicates a first order phase transition at $T=0$. The corresponding jump in the order parameter is shown in the lower panel of Fig. 3. At high temperatures, on the other hand, the order parameter decreases gradually to zero, indicating a second order phase transition. Thus, at the critical interaction strength and finite temperatures, the phase transition changes from first to second order.

For $J / W>0.55$, we do not find a CDW at $T=0$. Only at temperatures $T / W>0.004$, we can stabilize charge order. As we will show in Fig. 6, the charge ordered state possesses a gap at the Fermi energy. The region of chemical potential, in which the charge ordered state can be stabilized at quarter filling, is supposed to be related to the gap width, at least at $T=0$. This is because the state does not change when changing the chemical potential within this region. Therefore, the shrinking of the parameter region corresponds to a decrease of the gap width near the critical coupling strength. Furthermore, we see that the gap is closed for $J / W=0.6$ for $T / W<0.004$. Even if there would be a tiny region of chemical potential stabilizing charge order for $J / W \approx 0.6$ at $T=0$, the gap width of the state would be tiny and this state would be unstable towards perturbations. Thus, we believe that a reentrant behavior occurs for strong coupling strength in the vicinity of a first order phase transition.

Comparing to experiments, reasonable interaction strengths are $J / W<0.3$. If we assume a bandwidth of $W=1 \mathrm{eV}$ in our calculations, the resulting Kondo temperature as well as the transition temperature of the charge order for these interaction strengths are $T<$ $100 K$, which are realistic temperatures for $f$-electron systems.

J.E. Hirsch ${ }^{6}$ already pointed out that there exists a nearest-neighbor repulsive density-density interaction in second-order strong coupling expansion, which for weak coupling might be sufficient to drive the system into a CDW. However, the applicability of a strong coupling theory to explain the existence of the CDW in our calculations for small coupling strengths is questionable.

Another way of understanding the formation of a CDW at quarter filling is to consider the energy gain by the Kondo effect. In Fig. 4 we show the kinetic energy, $E_{k i n}$, and the energy of the exchange interaction, $J\langle\vec{S} \cdot \vec{s}\rangle$,
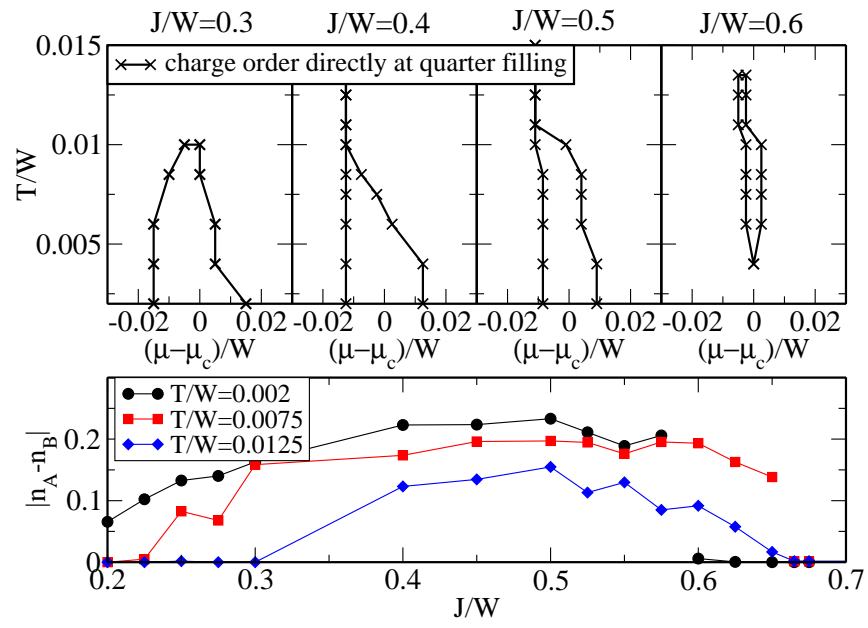

Figure 3: (Color online) Upper panels: Transition temperature over chemical potential for the CDW exactly at quarter filling. This plot illustrates the shrinking area of chemical potentials with increasing coupling strength, which stabilize the CDW at quarter filling. $\mu_{c}$ is the chemical potential at the center of the CDW phase at quarter filling. Lower panel: Charge order parameter for different interaction strengths.
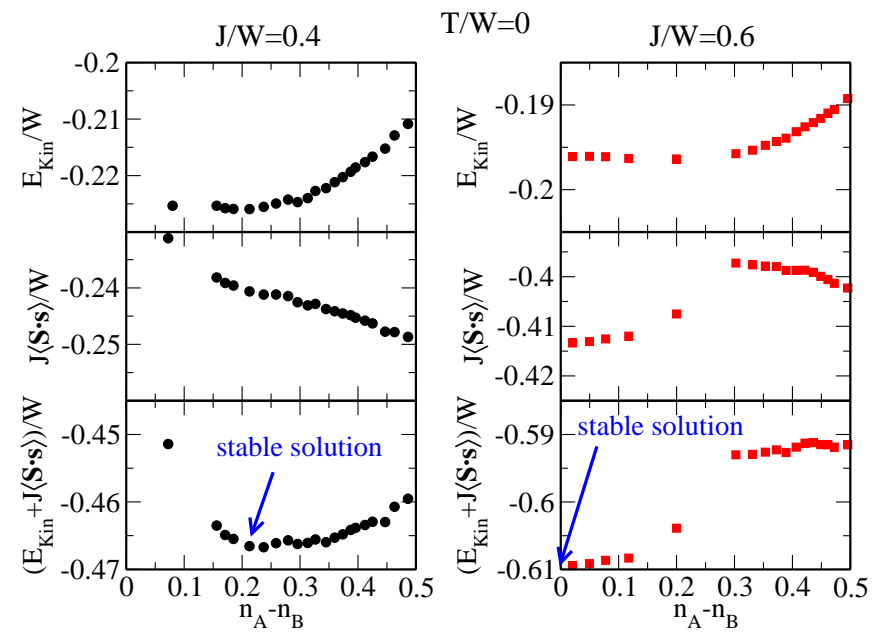

Figure 4: (Color online) Kinetic energy and $J\langle\vec{S} \cdot \vec{s}\rangle$ (coupling energy between localized spin and conduction electrons) for two different interaction values $J / W$. By introducing a staggered potential for the sublattices $\mathrm{A}$ and $\mathrm{B}$, we artificially create meta-stable solution with different occupation numbers, $n_{A}$ and $n_{B}$. For all solutions the system is kept at quarter filling.

for two coupling strengths calculated within DMFT at $T=0$. Starting from the converged DMFT solutions for both interaction values, we artificially change the magnitude of the charge order by introducing a staggered chemical potential. This staggered chemical potential increases or decreases the difference in the occupation numbers of sublattice A and B. For each obtained solution, we calculate the kinetic energy and the exchange energy. The calculated kinetic energies for both 
interaction values exhibit minima for $n_{A}=n_{B}$, corresponding to solutions without charge order. This can be expected, because neglecting the spin-electron coupling, the kinetic energy is supposed to drive the system into a paramagnetic state. On the other hand, the exchange energy shows a very interesting behavior. For $J / W=0.4$, which gives a CDW solution in Fig. 1, $J\langle\vec{S} \cdot \vec{s}\rangle$ decreases for increasing difference of the occupation numbers, $n_{A}-n_{B}$. Therefore, the exchange energy favors for $J / W=0.4$ a charge ordered state. The sum of kinetic energy and exchange energy results in a minimal energy for $n_{A}-n_{B} \approx 0.2$, which corresponds to the stable solution in our DMFT calculations without staggered potential. In contrast to $J / W=0.4$, the exchange energy for $J / W=0.6$ looks completely different. This energy has two local minima, one for a charge ordered state and the other for a state without charge order. The global minimum, and thus the energetically favored state, corresponds to the state without charge order. The sum of kinetic energy and exchange energy exhibits its global minimum for solutions without charge order, $n_{A}-n_{B}=0$.

Thus, we arrive at the conclusion that a change in the behavior of the exchange energy, $J\langle\vec{S} \cdot \vec{s}\rangle$, as a function of the particle number is responsible for driving the system into a charge ordered state. We can qualitatively explain this change by analyzing the Kondo energy, $E_{\text {Kondo }} \sim-W \exp \left(-1 /\left(J \rho_{\epsilon_{F}}\right)\right) \stackrel{29}{29}$ The Kondo energy depends via the DOS on the particle number. Thus, there might be parameter regions, where it is energetically favorable to have different electron occupations on sublattice A and B in order to gain Kondo energy. The importance of the Kondo effect and its dependence on the particle number is confirmed by the following two observations: DMFT calculations performed for the ferromagnetically coupled Kondo lattice model, $J<0$, at similar coupling strengths, do not show any sign of a CDW. Furthermore, DMFT calculations performed for the antiferromagnetically coupled Kondo lattice, $J>0$, but having a constant DOS, do also not show any sign of charge order. If the lattice has a constant DOS, the Kondo energy does not depend on the particle number, so that there is no energy gain by having different particle numbers on the two sublattices.

In Fig. 5 we show spectral properties of the CDW at $T=0$. The spectral functions of the CDW for both sublattices and for all coupling strengths $J$ possess a gap at the Fermi energy, thus being insulating. This could be expected due to the long range order and commensurate filling.

The qualitative shape of the spectrum can be understood in terms of a doped Kondo insulator. At half filling the Kondo effect splits the spectrum into two bands with a gap at the Fermi energy. For quarter filling, the Fermi energy is located at the center of the lower band. Because of the doubling of the unit cell due to the CDW, this lower band is again split into two bands with van Hove singularities at their band edges. This qualitative behav-

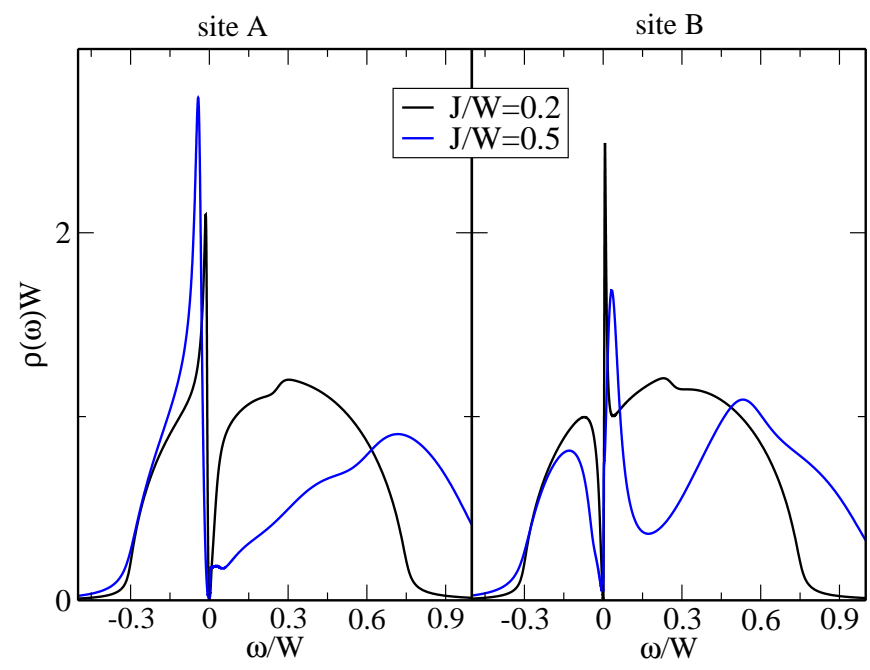

Figure 5: (Color online) Spectral function of the charge ordered state for A- and B-sublattice for two different coupling strengths. The Fermi energy corresponds to $\omega=0$.

ior can be explicitly seen for the $J / W=0.5$ curve in Fig. 5. The upper band of the Kondo insulator, which is unaffected by the charge order, is located at $\omega / W \approx 0.7$. The gap of the Kondo insulating phase corresponds to the dip located at $\omega / W \approx 0.2$. The lower band of the Kondo insulator, which is now split due to the CDW with van Hove singularities at the band edges, is located around the Fermi energy $\omega / W=0$. The charge order strongly affects the lower band of the Kondo insulator, which is filled differently on the two sublattices. For the smaller coupling strength $J / W=0.2$, the gap of the Kondo insulator is strongly smeared out, which makes the identification of these structures more difficult.

\section{COMPETITION BETWEEN FERROMAGNETISM AND CHARGE ORDER}

As already noted in the beginning, most previous studies found magnetism at quarter filling. Up to now we have completely suppressed magnetic ordering. In Fig. 6 we show the results of calculations simultaneously allowing for charge order and magnetism. In these calculations we allowed for any combination of magnetism and charge order on a two-site cluster. Thus, in principle also antiferromagnetic solutions would have been possible, but only ferromagnetic results turned out to be stable in our calculations. The left panel and the middle panel show the strength of the spin polarization and the charge order parameter, respectively. From this we draw a qualitative phase diagram in the right panel.

It should be noted that for these calculations we use mixing techniques within DMFT, 30 which help improving the convergence towards the solution. Furthermore, there are parameter regions for which the DMFT calculations without these mixing techniques at $T=0$ does 

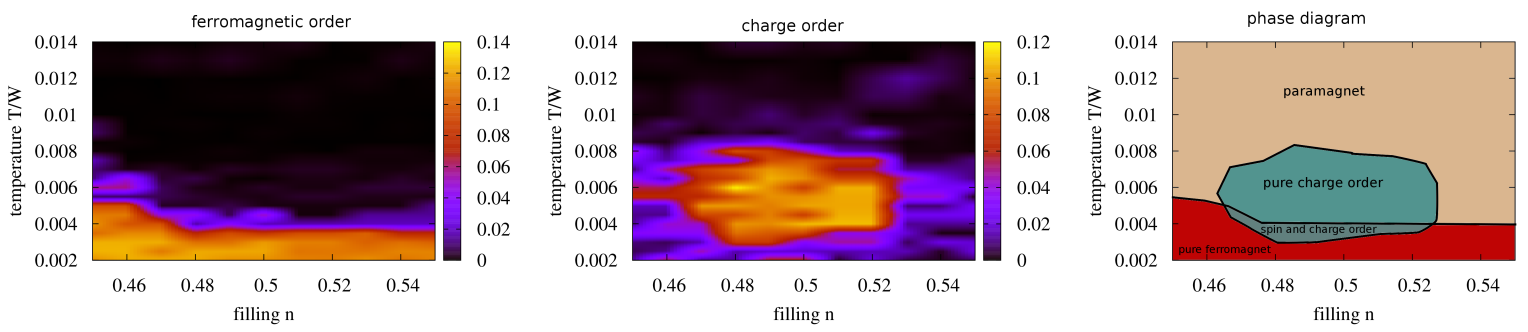

Figure 6: (Color online) Spin polarization (left panel) and charge order-parameter (middle panel) calculated from approximately 300 data points for $J / W=0.35$, simultaneously allowing for charge- and magnetic-order in two-site cluster. From these data the qualitative phase diagram (right panel) is drawn. The phase diagram comprises a pure ferromagnetic state at low temperatures, a transition region where charge and spin order can be found, a pure charge ordered state at intermediate temperatures, and a paramagnetic disordered state at high temperatures.

not seem to converge, but get stuck in oscillating solutions. This could indicate that there are other competing solutions, which however could not be stabilized.

From the panels showing the order parameters, it is clearly seen that the charge order parameter vanishes again at very low temperatures. The ground state is a magnetic (ferromagnetic) state without charge order. Thus, charge order and magnetic order are competing at $T=0$. However, charge order can be found when increasing the temperature. The magnetic state vanishes at a lower temperature compared to the transition temperature of the CDW. Therefore, exactly at quarter filling we will find a transition from a paramagnet to a charged ordered state which is followed by a region of coexistence between charge order and magnetism, when decreasing the temperature. Eventually at even smaller temperatures, this coexistence region vanishes, and a magnetic state without charge order is formed.

It should be noted that our calculations have been performed for an infinite dimensional Bethe lattice. Because of the simplicity of the Bethe lattice, i.e. there are no closed loops, order patterns involving more than two sites and combining magnetic- and charge-order are unlikely. Furthermore, we have also checked different high-dimensional lattice geometries like the hyper-cubiclattice, which exhibits a Gaussian DOS, and we found similar results as for the Bethe lattice. Thus, our results should be valid for bipartite lattices with similar DOS and large coordination number. We again want to stress that a lattice with constant DOS does not show charge order. Therefore, a curvature within the DOS is necessary. However, especially for low-dimensional lattices like the two dimensional square lattice, more complicated ground states allowing for a cooperation between charge order and magnetism might be favorable. In a recent study $\stackrel{31}{=}$ a combination of charge order and antiferromagnetism on four site clusters have been observed. Similar order patterns have also been observed for frustrated low dimensional lattices $\underline{\underline{8}} \underline{\underline{-12}}$ However, the existence of these states strongly depend on the lattice geometry, and these states are not easily generalized to arbitrary lattices for higher dimensions, $d>2$.

\section{CONCLUSIONS}

We have shown that charge order can exist in the Kondo lattice model without any repulsive interaction between different lattice sites or between $c$ - and $f$ - electrons. For this purpose we have used DMFT, which is exact in infinite dimensions, but is supposed to be a good approximation for three dimensional systems. We have shown that a paramagnetic charge ordered state exists for small coupling strengths, $J<0.65$ for a Bethe lattice, and vanishes as a first order phase transition for strong coupling. We have also shown that the charge ordered state at quarter filling is an insulator, as can be expected due to the doubling of the unit cell. As an explanation for the existence of charge order, the non-linear dependence of the Kondo energy is proposed.

Furthermore, we have studied the competition/cooperation of charge order and magnetic order. In these calculations we have found that the ferromagnetic state without charge order is the stable state at zero temperature, and charge order can be only found for increasing temperature when the magnetic state vanishes. However, in all our calculations we have seen that the CDW has the higher transition temperature compared to the transition temperature of the magnetic state. Thus, the experimentally observed charge order can be explained without any repulsive inter-site interactions, as long as the system is in the Kondo regime (odd number of f-electrons), as it is assumed for a Kondo lattice model.

Shortly before finishing this work, we became aware of

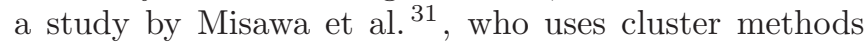
and Variational Monte Carlo for analyzing charge order in the Kondo lattice model. 


\section{Acknowledgments}

We acknowledge discussions with T. Yoshida and T Pruschke. RP thanks the Japan Society for the Promotion of Science (JSPS) for the support through its FIRST Program. SH acknowledges the Grant-in-Aid for JSPS Fellows. NK acknowledges support through KAKENHI
(No. 25400366 and 22103005), the Global COE Program "The Next Generation of Physics, Spun from Universality and Emergence" from MEXT of Japan, and JSPS through its FIRST Program. The numerical calculations were performed at the ISSP in Tokyo and on the SR16000 at YITP in Kyoto University.
* peters@scphys.kyoto-u.ac.jp

1 P. Coleman, Handbook of Magnetism and Advanced Magnetic Materials (John Wiley and Sons, 2007), p. 95.

2 P. Coleman and A. Schofield, Nature 433, 226 (2005).

3 P. Gegenwart and Q. Si, Nature Physics 4, 186 (2008).

4 S. Doniach, Physica B 91, 231 (1977).

5 Z. Hossaina, M. Schmidt, W. Schnelle, H. S. Jeevan, C. Geibel, S. Ramakrishnan, J. A. Mydosh, and Y. Grin, Phys. Rev. B 71, 060406(R) (2005).

6 J. Hirsch, Phys. Rev. B 30, 5383 (1984).

7 J. Otsuki, H. Kusunose, and Y. Kuramoto, J. Phys. Soc. Jpn. 78, 034719 (2009).

8 Y. Motome, K. Nakamikawa, Y. Yamaji, and M. Udagawa, Phys. Rev. Lett. 105, 036403 (2010).

9 S. Hayami, M. Udagawa, and Y. Motome, J. Phys. Soc. Jpn. 80, 073704 (2011).

10 S. Hayami, M. Udagawa, and Y. Motome (2012), arXiv:1208.4691.

11 H. Ishizuka and Y. Motome, Phys. Rev. Lett. 108, 257205 (2012).

12 Y. Akagi and Y. Motome (2012), arXiv:1206.0374.

13 C. Lacroix and M. Cyrot, Phys. Rev. B 20, 1969 (1979).

14 P. Fazekas and E. Muller-Hartmann, Z. Phys. B: Condens. Matter 85, 285 (1991).

15 A. Georges, G. Kotliar, W. Krauth, and M. Rozenberg, Rev. Mod. Phys. 68, 13 (1996).

16 T. Pruschke, M. Jarrell, and J. Freericks, Adv. Phys. 44,
187 (1995).

17 K. Wilson, Rev. Mod. Phys. 47, 773 (1975).

18 R. Bulla, T. Costi, and T. Pruschke, Rev. Mod. Phys. 80, 395 (2008).

19 R. Peters, T. Pruschke, and F. Anders, Phys. Rev. B 74, 245114 (2006).

20 A. Weichselbaum and J. von Delft, Phys. Rev. Lett. 99, 076402 (2007).

21 M. Jarrell, H. Akhlaghpour, and T. Pruschke, Phys. Rev. Lett. 70, 1670 (1993).

22 S.-J. Sun, M.-F. Yang, and T.-M. Hong, Phys. Rev. B 48, 16127 (1993).

23 M. J. Rozenberg, Phys. Rev. B 52, 7369 (1995).

24 P. Sun and G. Kotliar, Phys. Rev. Lett. 95, 016402 (2005).

25 R. Peters and T. Pruschke, Phys. Rev. B 76, 245101 (2007).

26 L. De Leo, M. Civelli, and G. Kotliar, Phys. Rev. B 77, 075107 (2008).

27 S. Hoshino, J. Otsuki, and Y. Kuramoto, Phys. Rev. B 81, 113108 (2010).

28 R. Peters, N. Kawakami, and T. Pruschke, Phys. Rev. Lett. 108, 086402 (2012).

29 A. Hewson, The Kondo Problem to Heavy Fermions (Cambridge University Press, 1997).

30 R. Žitko, Phys. Rev. B 80, 125125 (2009).

31 T. Misawa, J. Yoshitake, and Y. Motome (2013), arXiv:1303.3168. 